\title{
PERFORMANCE OF A LINEAR INTERFERENCE CANCELLER FOR A DS/ CDMA SYNCHRONOUS SYSTEM BASED ON THE EKF DELAY ESTIMATOR
}

\author{
Pilar Díaz, Ramón Agustí \\ Dept. of Signal Theory and Communications \\ Universitat Politècnica de Catalunya \\ C/ Sor Eulàlia d'Anzizu s/n Mòdul D4 \\ 08034 Barcelona, Spain \\ Tel. 34-3-4017201, Fax 34-3-4017200 \\ e-mail: pilar@xaloc.upc.es
}

\begin{abstract}
Since the conventional detector often fails to produce reliable decisions for a CDMA channel, several new multiuser detectors have been proposed lately. In this paper, we propose both a simple linear scheme for interference cancellation, which exhibits good performance, and a synchronism scheme based on the Extended Kalman Filter (EKF) to achieve synchronization among the different users at the base station, for a directsequence code-division multiple-access (DS/CDMA) synchronous system. The influence of the synchronism scheme on the performance of the interference canceller is analyzed under Rayleigh multipath fading.
\end{abstract}

\section{INTRODUCTION}

In mobile communication systems of next generation, the increase in capacity will be one of the most important requirements to be fulfilled. In order to improve the capacity in a DS/CDMA cellular system, which is basically limited by interference, known information on interfering signals can be employed to eliminate them at the base station. The optimum demodulator for signals that share the same channel in time and frequency, described in [1], is based on a multiuser joint detection scheme. This optimum receiver is, however, too complex to be implemented in a real mobile communication system, since its grows exponentially in complexity as the number of simultaneous users to be detected increases.
An alternative scheme based on interference cancellation techniques is presented here for a synchronous DS/CDMA cellular system, which employs Gold codes as spreading sequences. The use of quasi-orthogonal sequences as the Gold codes in a synchronous DS/CDMA system improves the performance obtaiued with an asynchronous access as a result of the good behaviour of these sequences in terms of periodic cross-correlation. Nevertheless, in a multipath environment, the quasi-orthogonality between users in the same cell is destroyed by the multiple propagation paths coming at the receiver. In order to keep on this quasiorthogonality, it is necessary to resort to a cancellation scheme that eliminates the interfering propagation paths, as shown in Fig. 1. In this case, the complexity of the receiver only grows linearly as the number of simultaneous users within a given cell increases. Despite its simplicity, it is shown in [2] via some computer simulation that this scheme improves significantly the behaviour of the conventional

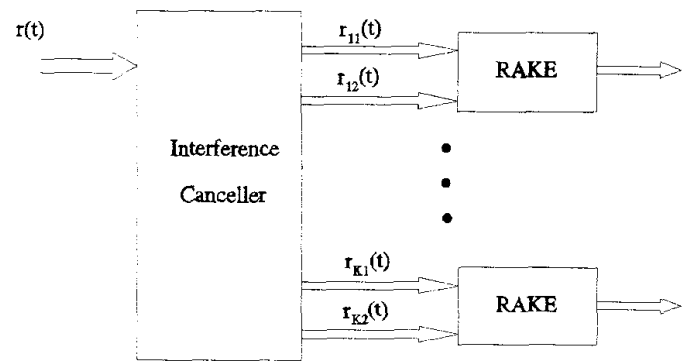

Fig. 1. A DS/CDMA receiver with an interference cancellation scheme. 
receiver in terms of capacity.

To support this synchronous access among the different mobiles at the base station, a simple procedure based on a delay estimation scheme of low complexity is illustrated in Fig. 2. The procedure we propose consists of estimating the channel delay of every mobile at the base station, and according to the time reference retained at the base, send the appropriate command to each mobile in order to make them adjust their transmission time to this reference. If this adjustment is done in steps of $\Delta$ seconds, let us note that the synchronism command to be sent to every user would be made up of only one bit.

The synchronism scheme this procedure is based on consists of a channel delay estimator based on the EKF, which is illustrated in Fig. 3., and designed for working properly in tracking [3].

Let $r(t)$ be the received signal, $c(t)$ the spreading code assigned to the user of interest and $\hat{\tau}_{\mathrm{r}}$ the channel delay to be estimated. The first block of the delay estimator processes the received signal in order to improve the signal-tointerference power ratio, extremely low in CDMA environments. The second block is the EKF, which is responsible for the estimation of the delay introduced by the mobile channel.

\section{DESCRIPTION OF THE CANCELLATION SCHEME}

Fig. 4 shows the interference cancellation scheme proposed for a synchronous DS/CDMA system: cancellation is carried out before combining the different propagation paths at the Rake receiver.

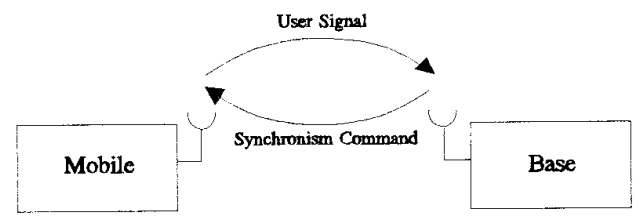

Fig. 2. Synchronism scheme for a DS/CDMA synchronous system.

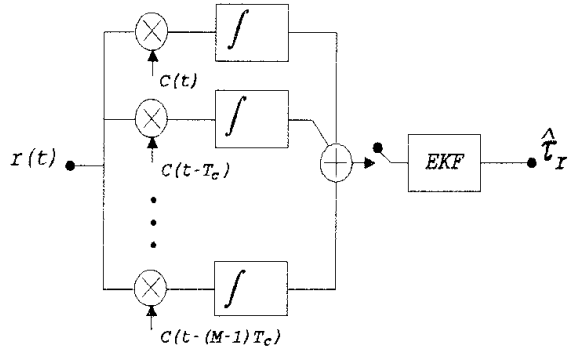

Fig. 3. Synchronism scheme based on the Extended Kalman Filter.

Let $c_{k}(t)$ be the Gold sequence assigned to the $k$-th user, $L$ the number of propagation paths at the receiver input, $M$ the number of arms at the Rake receiver, $K$ the number of simultaneous users in a given cell and $S$ the average received power in absence of multipath. The "user k estimator" in Fig. 4 computes $s_{\mathrm{kn}}(\mathrm{t})(\mathrm{n}=0, \ldots, \mathrm{L}-1)$ from the received signal, $\mathbf{r}(\mathrm{t})$ :

$$
s_{k n}(t)=\sum_{i=-\infty}^{\infty} \sqrt{2 S} \hat{\alpha}_{k n} c_{k}\left(t-n T_{c}+\hat{\tau}_{r k}\right) \hat{d}_{k}^{i} r e c t_{T_{t}}\left(t-i T_{b}+\hat{t}_{r k}\right)
$$

where $\hat{\alpha}_{\mathrm{kn}}$ and $\hat{\mathrm{d}}_{\mathrm{k}}^{\mathrm{j}}$ are respectively the estimate of the $\mathrm{n}$-th propagation path complex amplitude and the estimate of the i-th transmitted bit, both corresponding to the $\mathrm{k}$-th user, and rect $_{T}(t)$ represents a square waveform of duration $T$.

Let $n(t)$ represent the interference generated by other users in the adjacent cells. When perfect synchronism between users within the same cell and perfect estimates of the channel impulsional response and transmitted bits are assumed, it can be shown that $r_{j \mathrm{~m}}(t)(j=1, \ldots, K ; m=0, \ldots, M-$ 1) after cancellation takes the expression [2]:

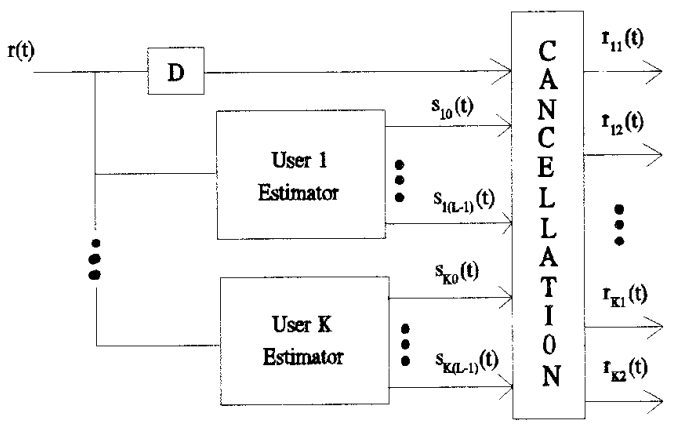

Fig. 4. Block diagram of the interference canceller. 


$$
r_{j m}(t)=\sum_{i=-\infty}^{\infty} \sqrt{2 S} \alpha_{j m} c_{j}\left(t-m T_{\partial}\right) d_{j}^{i} \operatorname{rect}_{T_{b}}\left(t-i T_{b}\right)+n(t)
$$

That is, in a cellular mobile system limited by interferences, a linear canceller such as this proposed here would be ideally limited in capacity by the interfering signals coming from adjacent cells.

In practice, however, perfect estimates are not available and perfect synchronism between users cannot be accomplished so, throughout some extensive simulations, we will show in section IV that the performance of the canceller scheme in a real environment lies very closed to this of the ideal case.

\section{SimUlATION MODEL}

In order to assess the performance of the proposed cancellation scheme for a DS/CDMA CBPSK synchronous system based on the EKF delay estimator, the complete transmission chain has been simulated for $\mathrm{K}$ different users within a same cell. Such a chain is shown in Fig. 5, where $s_{T K}(t)$ represents the complex envelope of the signal transmitted by the $\mathrm{k}$-th mobile:

$$
s_{T k}(t)=\sum_{i=-\infty}^{\infty} \sqrt{2 S} c_{k}(t) d_{k}^{i} r e c t_{T_{b}}\left(t-i T_{b}\right)
$$

The set of Gold codes assigned to each user, $c_{k}(t)$ $(\mathrm{k}=1, \ldots, \mathrm{K})$, has been obtained from two PN sequences of length $N=127$ chips, generated from the characteristic polynomials 211 and 277 (octal) respectively [4].

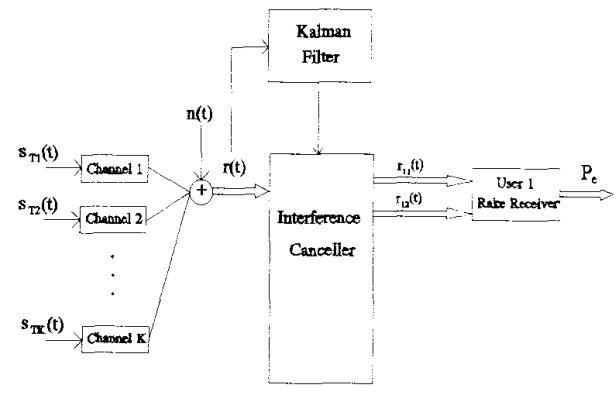

Fig. 5. Block diagram of the simulated DS/CDMA synchronous system
Let $h_{k}(t)$ be the low-pass equivalent impulsional response of the channel for the $\mathrm{k}$-th user:

$$
h_{k}(t)=\sum_{n=0}^{t-1} \alpha_{k n} \delta\left(t-n T_{c}+\tau_{r k}\right)
$$

The channel has been modelled with an exponential Power Delay Profile (PDP), with a Delay Spread normalised to the bit interval equal to $\mathrm{D}_{\mathrm{sn}}=0.01$, as usual in an indoor environment.

Concerning the low-pass equivalent Doppler Power Spectrum of each channel propagation path, the model "class" [5], given by the expression

$$
S(f)=\frac{A}{\sqrt{1-\left(\frac{f}{f_{d}}\right)^{2}}} \quad-f_{d}<f<f_{d}
$$

has been assumed, where $f_{d}$ is the maximum Doppler frequency. $f_{d} T_{b}=0.0005$ has been set in our simulations to model the channel variations in an indoor environment.

Finally, concerning computation of the bit and the channel impulsional response, which are needed to perform cancellation, a Rake receiver and a filter matched to the corresponding Gold sequence have been considered respectively. A data frame has been designed in order to allow for estimation of the channel impulsional response during the reception of the known bits in the preamble, as shown in Fig. 6, and estimation of the transmitted bits during the reception of the information block. Q and $D$ have been dimensioned for an indoor environment $(Q=10$ bits and $\mathrm{D}=90$ bits) according to [6].

\section{RESULTS AND CONCLUSIONS}

The performance of the linear interference canceller has been analyzed under the assumption of an open-loop power control and an EKF-based synchronism. Extensive simulations of the whole system have allow us to study the canceller behaviour under realistic situations in terms of capacity and analyze the EKF-based delay estimator influence 


\begin{tabular}{|c|c|}
\hline Preamble & Information \\
\hline$Q$ bits & D bits \\
\hline
\end{tabular}

Fig. 6. Data frame structure employed in the simulation of the system.

on the canceller performance.

The mean bit error tate for a fixed number of simultaneous users has been taken as figure of merit to evaluate system performance. Some representative figures have been built from the results we have obtained in our simulations. Among these figures, we have chosen Fig. 7 and Fig. 8 that illustrate the mean BER in terms of the system capacity for an ideal synchronous access and for a synchronous access based on the EKF delay estimator. The number of arms in the Rake receiver has been assumed to be $M=2$ in both cases.

From the two of them, we can conclude that the use of the canceller structure results in an important capacity improvement with regard to the single-user receiver, i.e. a simple Rake receiver, which may be even greater in the presence of channel encoding. In addition, the synchronism scheme based on the EKF is robust enough with very little impact on the canceller performance. This is the reason why

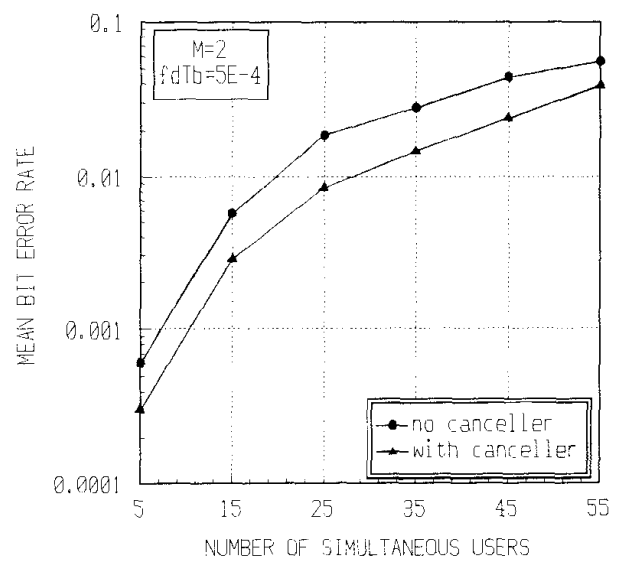

Fig. 7. Bit Error Rate at the output of the canceller for an ideal synchronism.

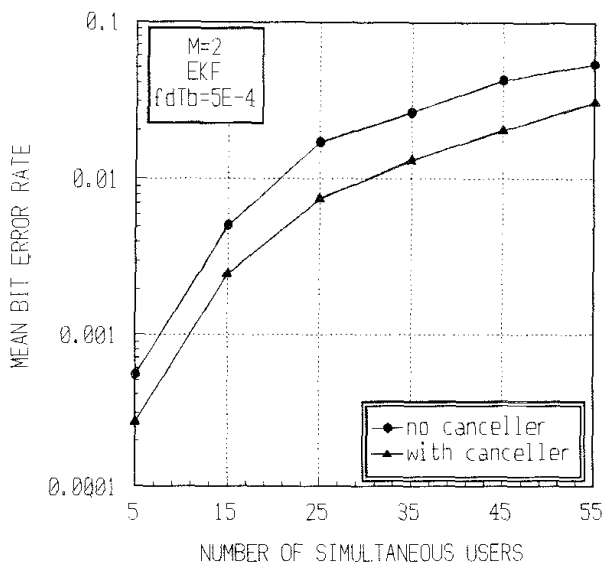

Fig. 8. Bit Error Rate at the output of the canceller for an EKF-based synchronism.

it seems to be a good candidate for synchronising the different users at the base station of a synchronous system in Rayleigh multipath environments.

\section{REFERENCES}

[1] S. Verdú, "Minimum Probability of Error for Asynchronous Gaussian Multiple-Access Channels", IEEE Trans. on Information Theory, Vol. IT-32, January 1986.

[2] P. Díaz, R. Agustí, "Analysis of a Linear Interference Canceller in Cellular DS/CDMA Systems", in Proceedings of the 43rd IEEE VTC, June 1994.

[3] P. Díaz, D. Henche, R. Agustí, "A PN Code Delay Estimator Based on the Extended Kalman Filter for a DS/CDMA Cellular System", in Proceedings of the Ath International Symposium on PIMRC, September 1993

[4] R. de Gaudenzi, C. Elia, R. Viola, "Bandlimited Quasi-Synchronous CDMA: A Novel Satellite Access Technique for Mobile and Personal Communication Systems", IEEE Journal on Selected Areas in Communications, Vol. 10, No. 2, February 1992.

[5] Cost 207, "Digital Land Mobile Radio Communications", Information Technologies and Sciences (CEC), 1989.

[6] P. Díaz, R. Agustí, "On the Influence of the Channel Impulsional Response Estimation on the Performance of a Linear Interference Canceller for a DS/CDMA System", in Proceedings of the 5th International Symposium on PIMRC, September 1994. 